\title{
Hepatosplenic Candidiasis
}

\author{
Oliver A. Cornely M.D., ${ }^{*}$ Christopher Bangard M.D., ${ }^{\dagger}$ and Natalie I. Jaspers M.D. ${ }^{\neq}$
}

Hepatosplenic candidiasis is a very rare disease, but is the most frequent form of chronic disseminated candidiasis. It typically occurs in patients with long-lasting neutropenia defined by more than 10 days of absolute neutrophil counts (ANC) below 500/ $\mathrm{LL}$, eg, patients undergoing remissioninduction chemotherapy for acute leukemia. ${ }^{1}$ In a large $(\mathrm{N}=602)$ randomized double-blind clinical trial on antifungal prophylaxis in acute myelogenous leukemia, the breakthrough rate of invasive candidiasis was $0.8 \%$. All of these cases were candidemic, ie, acute disseminated candidiasis, and not a single patient developed chronic disseminated candidiasis. ${ }^{2}$ Acute and chronic disseminated candidiasis are differentiated by the presence or absence of sepsis syndrome. ${ }^{3}$ Since the clinical trial mentioned above, use of triazole antifungal prophylaxis expanded and likely led to a further decrease of the incidence rate. However, patient groups who cannot receive azoles, eg, due to drugdrug interaction with vinca alkaloids in acute lymphoblastic leukemia, will continue to be at risk for hepatosplenic candidiasis, in particular if treated with extended courses of glucocorticosteroids. ${ }^{1,4}$

Although many, if not all patients with long-lasting neutropenia will experience at least one febrile episode, any fever usually ends when ANC recovers. A persisting fever after ANC recovery, and in particular fever just beginning with ANC recovery in a previously afebrile patient, should prompt evaluation for hepatosplenic candidiasis, although other causes may be present. Additional symptoms supporting the suspicion of hepatosplenic candidiasis are as unspe- cific as abdominal pain, diarrhea, tenderness, nausea, vomiting, and sometimes jaundice. In the largest series published to date, the latter was present in less than one-third of patients. ${ }^{1}$ Clinical chemistry may show a pattern of more than three-fold increased serum alkaline phosphatase with other liver function tests, in particular transaminases and gamma-glutamyl-transferase being less sensitive. ${ }^{1}$

In hepatosplenic candidiasis, frequently no fungus is isolated from blood cultures. ${ }^{1}$ A potential explanation for the low blood culture yield may be hematogenous dissemination limited to the portal vein system. The majority of fungal species involved in cases with positive blood culture were C. albicans, C. tropicalis, and C. parapsilosis, thus resembling a common distribution of pathogens colonizing patients with hematological disease. ${ }^{1}$ In the individual patient, one extrapolates colonization results to estimate the species causing invasive disease. ${ }^{5}$ In a series from Italy, twothirds of cases had Candida-positive biopsies. ${ }^{1}$ However, histology and fungal cultures are often negative in our experience. Thus, hepatosplenic candidiasis is one of the few infections where we actually prefer to treat rather than diagnose. It has been hypothesized that proof of fungal tissue invasion often remains elusive, because the clinical syndrome is a mixture of fungal abscesses and immune reconstitution inflammatory syndrome. ${ }^{6}$ If both aspects were consecutive processes, timing of biopsy would be important. Because in the majority of patients with hepatosplenic candidiasis, the disease becomes clinically apparent only with resolving neutropenia and thrombocytopenia, we may

Abbreviations: ANC, absolute neutrophil count; CEUS, contrast-enhanced ultrasound; CT, computed tomography; MRI, magnetic resonance imaging From the *Cologne Excellence Cluster on Cellular Stress Responses in Aging-Associated Diseases (CECAD), Department I of Internal Medicine, Clinical Trials Centre Cologne, ZKS Köln, Center for Integrated Oncology CIO KöInBonn, German Centre for Infection Research, University of Cologne, Cologne, Germany

${ }^{+}$Department of Radiology, University of Cologne, Cologne, Germany, and

${ }^{\ddagger}$ Department of Gastroenterology and Hepatology, University of Cologne, Cologne, Germany

Additional Supporting Information may be found in the online version of this article.

Potential conflict of interest: Nothing to report.

View this article online at wileyonlinelibrary.com

(C) 2015 by the American Association for the Study of Liver Diseases

doi: 10.1002/cld.491 


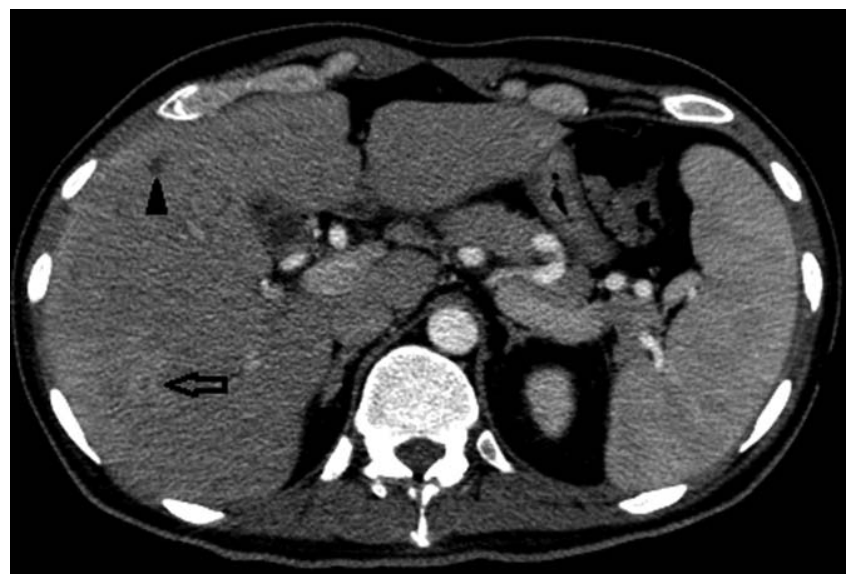

Figure 1 Arterial phase CT with "bull's eye lesion" (arrow) and a hypodense lesion without rim enhancement (arrowhead).

systematically biopsy too late. If chronic disseminated candidiasis is impossible to prove by microbiological evidence, imaging is of particular importance to define probable hepatosplenic candidiasis. ${ }^{3}$ Clinically, a fever as described above will trigger thoracic and abdominal computed tomography (CT) scans and follow-up examinations by ultrasound.

CT or magnetic resonance imaging (MRI) scans detect hepatosplenic candidiasis rarely before bone marrow reconstitution and ANC recovery. Lesion enhancement pattern differs for acute, subacute, and chronic stage of disease. The most sensitive CT phase for liver involvement in the acute stage is the arterial dominant phase, ie, 25-35 seconds after contrast injection, showing a hyperattenuating rim surrounding a hypoattenuating center "bull's eye" (Fig. 1) or a hyperattenuating lesion. ${ }^{7}$ In the portal-venous phase, ie, 60-80 seconds after injection, microabscesses are very often hypoattenuating lesions of $\leq 1 \mathrm{~cm}$ size (Fig. 2, Fig. 3, and

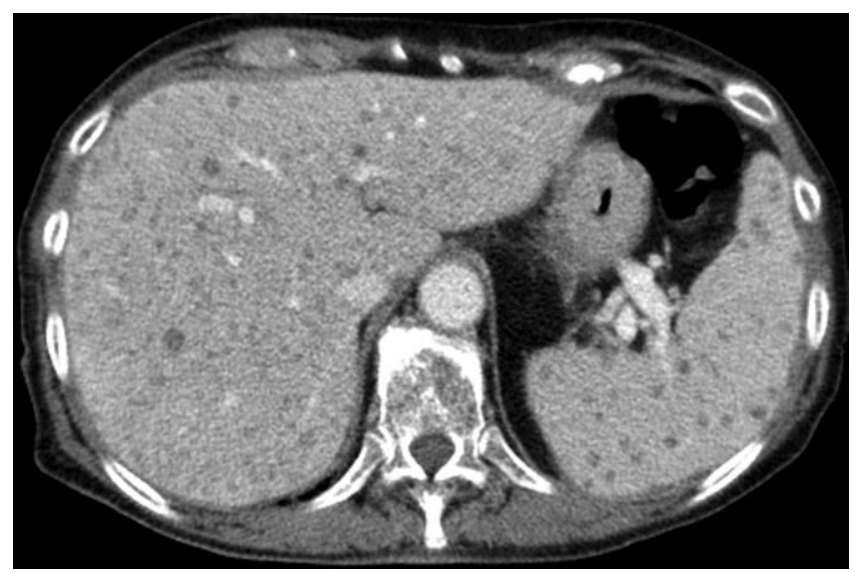

Figure 2 Portal venous phase CT with multiple hypodense Candida microabscesses in the liver and spleen.

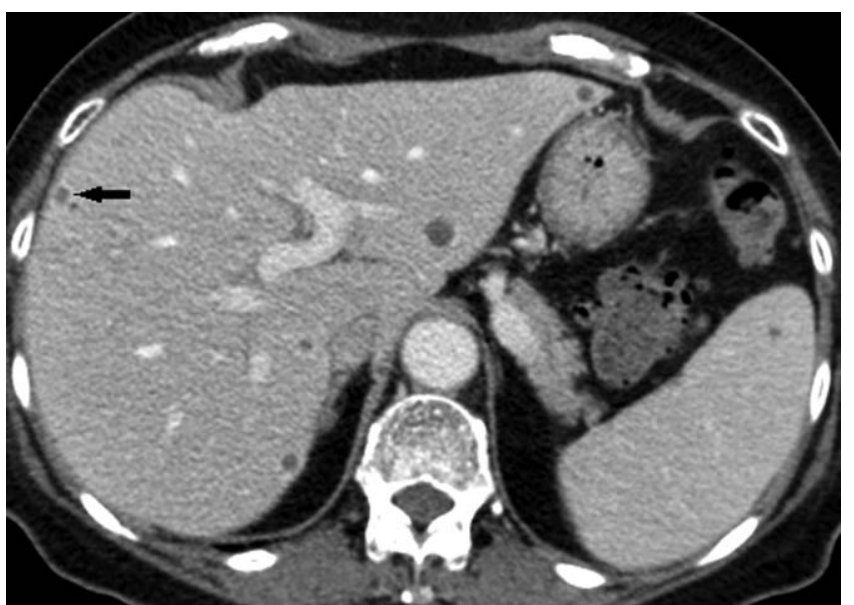

Figure 3 Portal venous phase CT with multiple hypodense Candida microabscesses in the liver and spleen. Note peripheral contrast uptake of the small abscess (arrow) which is often not detectable in microabscesses.

Video 1). Both phases are more sensitive than nonenhanced images. Initially hypodense lesions in the acute phase on nonenhanced CT (Fig. 4) can become hyperdense by hemorrhage or calcification in the late subacute or chronic stage (Fig. 5). Dynamic contrast-enhanced MRI is superior to portal venous phase $\mathrm{CT}^{8}$ and comparable to biphasic arterial dominant and portal venous phase CT. Because the standard work-up of febrile neutropenic patients includes nonenhanced thoracic CT, imaging of the liver in suspected hepatosplenic candidiasis is mostly done by contrastenhanced biphasic CT and not by MRI.

Upon ultrasound analysis, chronic disseminated candidiasis does not usually demarcate before ANC recovery. In gray-scale ultrasound, multiple round lesions measuring 5-20 $\mathrm{mm}$ are scattered throughout the parenchyma of liver, spleen, and sometimes kidney (Video 4a). The most

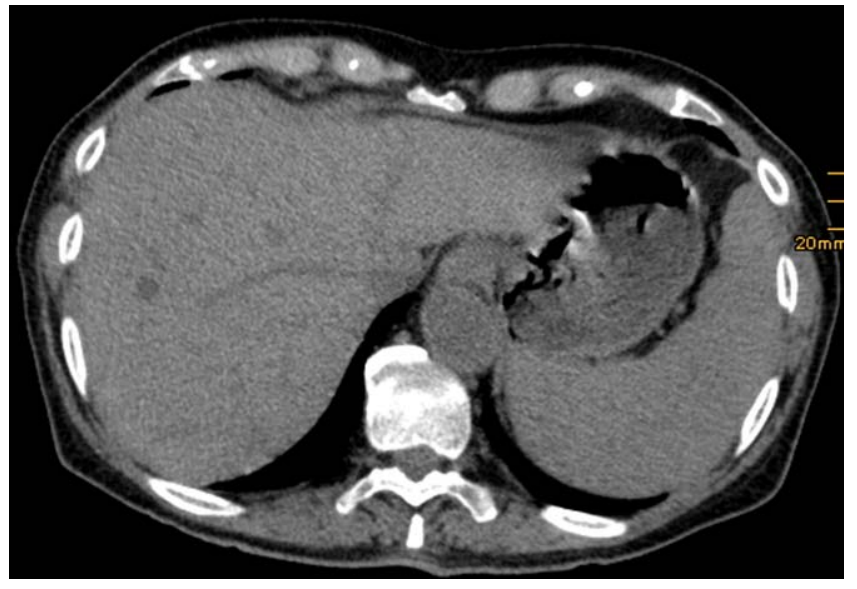

Figure 4 Nonenhanced CT with one hypodense abscess in the early stage. 


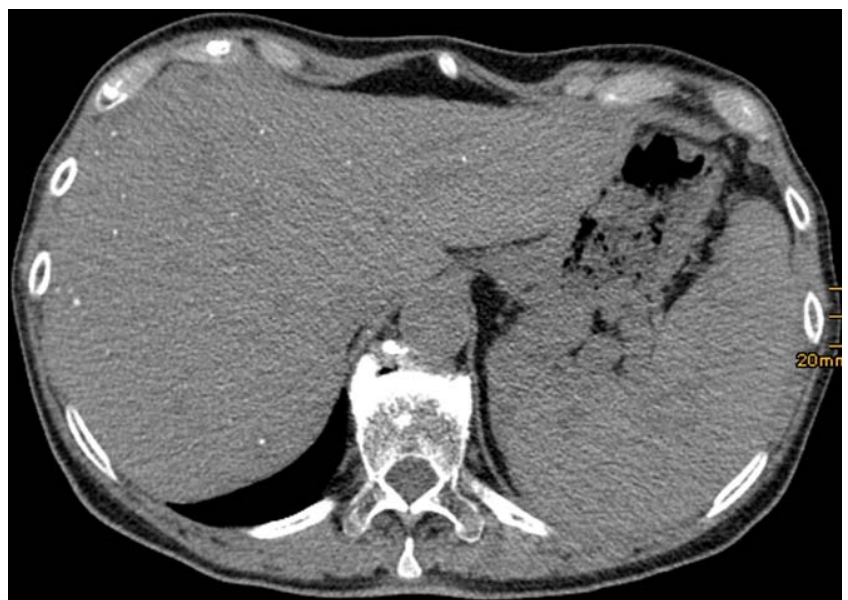

Figure 5 Nonenhanced CT with multiple tiny calcifications of the abscesses after antifungal therapy in the liver. Note an enlarged spleen with hypodense lesions.

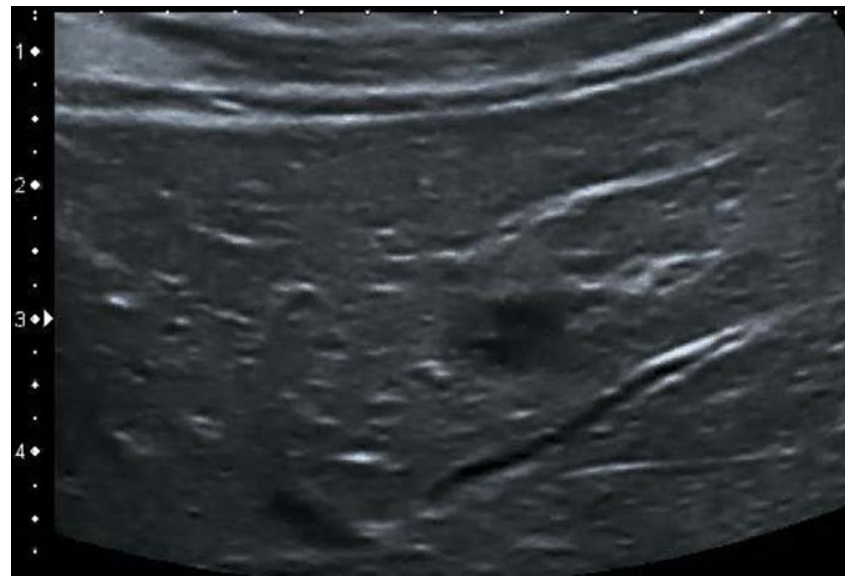

Figure 6 Hypoechoic small lesions with a maximum diameter of $20 \mathrm{~mm}$.

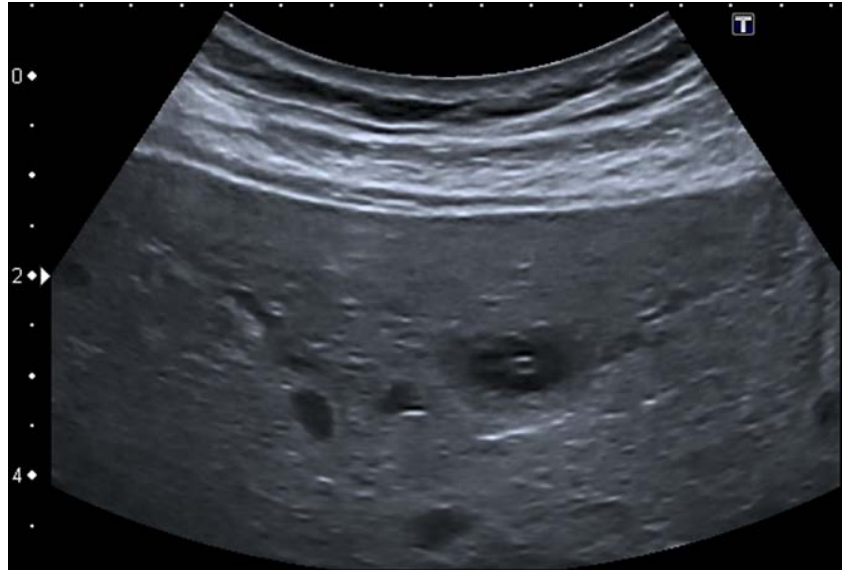

Figure 7 Typical bull's eyes or target pattern with a peripheral hypoechoic halo encircling a central hyperechoic core.

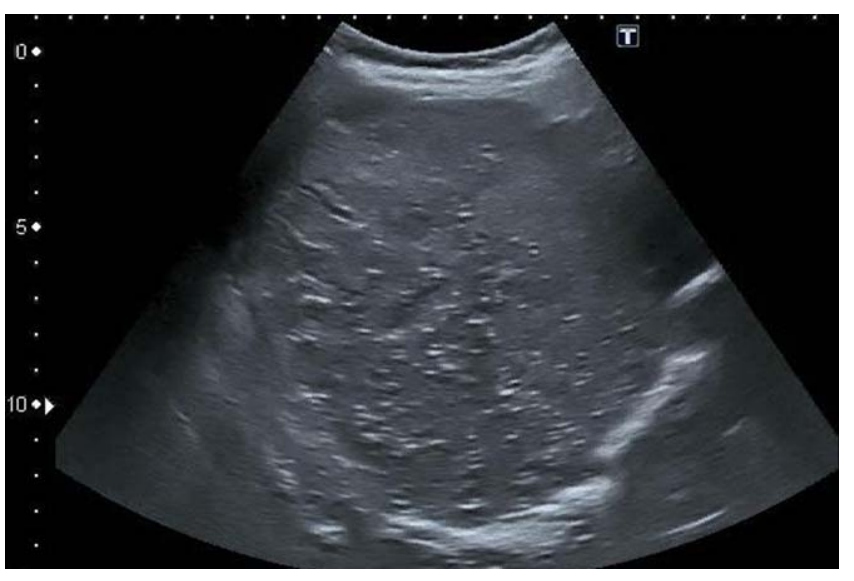

Figure 8 Hyperechoic lesions with dorsal shadowing in a late stage.

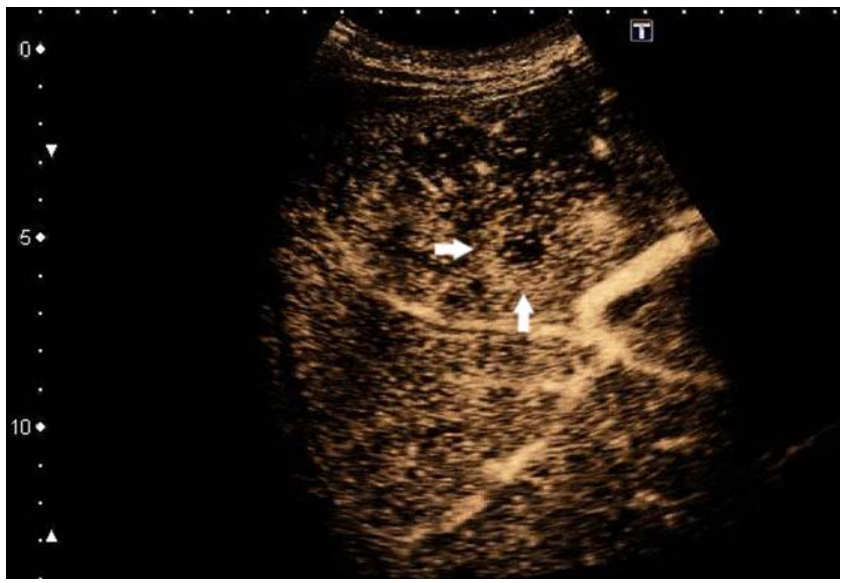

Figure 9 Hyperechoic rim enhancement with a central hypoechoic area.

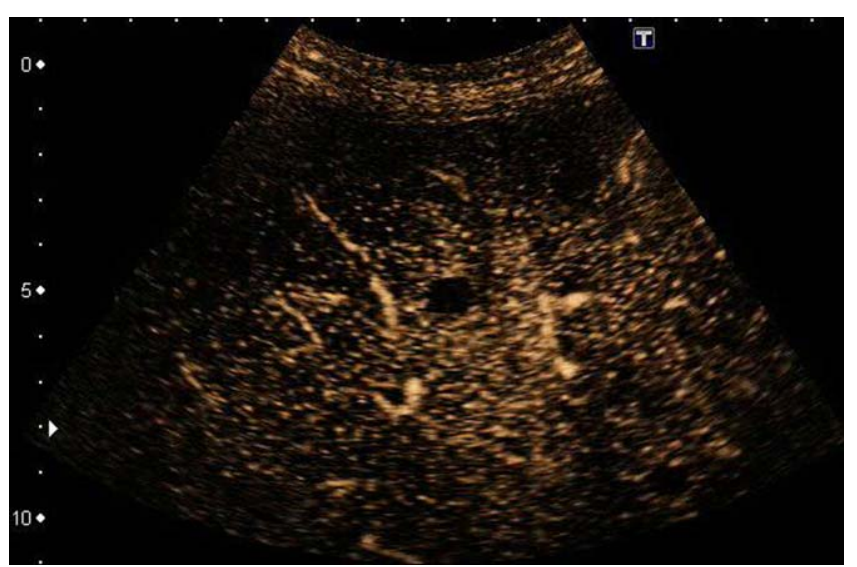

Figure 10 Isoechoic rim enhancement with a central hypoechoic area. 
frequent pattern is disseminated hypoechoic small lesions (Fig. 6). Difficult-to-differentiate diagnoses are lymphoma of the liver, leukemic infiltrates, and metastases. Similar to CT, a typical ultrasound phenotype of hepatic candidiasis is the bull's eye or target pattern with a peripheral hypoechoic halo encircling a central hyperechoic core (Fig. 7). In the late stage, hyperechoic lesions with dorsal shadowing may be seen (Fig. 8). ${ }^{9}$ Occasionally, hepatosplenic candidiasis may not be detectable with B-mode ultrasound. Contrastenhanced ultrasound (CEUS) with sulfur hexafluoride contrast (SonoVue, Bracco International, Amsterdam, The Netherlands) shows a late-phase washout, ie, 90-240 seconds after injection (Videos 2c, 4b, and 5). In the arterial phase, ie, 5-25 seconds after injection, lesions often have a hyperechoic rim enhancement with a growing central washout in the portal-venous and late phase (Fig. 9, Video 3). Sometimes, the lesions exhibit an isoechoic rim enhancement (Fig. 10, Video 2a and 2b); others may be hypoechoic in all contrast phases without any rim enhancement. ${ }^{10}$ Advantages of CEUS are absence of nephrotoxicity and radiation exposure, and the feasibility of a bedside examination.

Suspected diagnosis on the basis of clinical picture and imaging results should prompt treatment. The recent Euro-

\section{References}

1. Pagano L, Mele L, Fianchi L, Melillo L, Martino B, D'Antonio D, et al. Chronic disseminated candidiasis in patients with hematologic malignancies. Clinical features and outcome of 29 episodes. Haematologica 2002;87:535-541.

2. Cornely OA, Maertens J, Winston DJ, Perfect J, Ullmann AJ, Walsh TJ, et al. Posaconazole vs. fluconazole or itraconazole prophylaxis in patients with neutropenia. N Engl J Med 2007;356:348-359.

3. de Pauw B, Walsh TJ, Donnelly JP, Stevens DA, Edwards JE, Calandra T, et al. Revised definitions of invasive fungal disease from the European Organization for Research and Treatment of Cancer/Invasive Fungal Infections Cooperative Group and the National Institute of Allergy and Infectious Diseases Mycoses Study Group (EORTC/MSG) Consensus Group. Clin Infect Dis 2008;46:1813-1821.

4. Tacke D, Buchheidt D, Karthaus M, Krause SW, Maschmeyer G, Neumann S, et al. Primary prophylaxis of invasive fungal infections in patients with haematologic malignancies. 2014 update of the recommendations of the Infectious Diseases Working Party of the German Society for Haematology and Oncology. Ann Hematol 2014;93:1449-1456.

5. Pagano L, Antinori A, Ammassari A, Mele L, Nosari A, Melillo L, et al. Retrospective study of candidemia in patients with hematological malignancies. Clinical features, risk factors and outcome of 76 episodes. Eur J Haematol 1999;63:77-85.

6. Rammaert B, Desjardins A, Lortholary O. New insights into hepatosplenic candidosis, a manifestation of chronic disseminated candidosis. Mycoses 2012;55:e74-e84 pean Society of Clinical Microbiology and Infectious Diseases guideline recommends fluconazole, and the most frequent isolates C. albicans, C. tropicalis, and C. parapsilosis are usually susceptible to that agent. Treatment experience is largely anecdotal. ${ }^{11}$ At our hospital, we recommend fluconazole $400 \mathrm{mg} /$ day if documented or presumed susceptibility and liver function tests allow. Liposomal amphotericin B and echinocandin antifungals are alternatives. ${ }^{1,12}$ Treatment usually lasts 3 months or longer, so that oral treatment is preferable. Following the hypothesis that chronic disseminated candidiasis is driven by immune reconstitution inflammatory syndrome, concomitant glucocorticosteroids have been prescribed successfully in a limited number of patients. Doses of $\geq 5 \mathrm{mg} / \mathrm{kg}$ prednisone equivalent for at least 3 weeks led to rapid resolution of symptoms. ${ }^{13}$ Consensus criteria for glucocorticosteroid dose and for the appropriate time point to initiate steroid treatment have not been developed.

\section{CORRESPONDENCE}

Oliver Cornely •••. Email: Oliver.Cornely@zks-koeln.de

7. Metser U, Haider MA, Dill-Macky M, Atri M, Lockwood G, Minden M. Fungal liver infection in immunocompromised patients: depiction with multiphasic contrast-enhanced helical CT. Radiology 2005;235:97-105.

8. Anttila VJ, Lamminen AE, Bondestam S, Korhola O, Farkkila M, Sivonen A, et al. Magnetic resonance imaging is superior to computed tomography and ultrasonography in imaging infectious liver foci in acute leukaemia. Eur J Haematol 1996;56:82-87.

9. Wermke W. Sonographische Differenzialdiagnose Leberkrankheiten. Cologne, Germany: Deutscher Ärzte-Verlag; 2006.

10. Görg C, Bert T, Klassen E, Neesse A, Barth P, Neubauer A. Contrast enhanced sonographic patterns of hepatic candidiasis [in German]. Z Gastroenterol 2010;48:678-682.

11. Ullmann AJ, Akova M, Herbrecht R, Viscoli C, Arendrup MC, Arikan-Akdagli $\mathrm{S}$, et al. ESCMID guideline for the diagnosis and management of Candida diseases 2012: adults with haematological malignancies and after haematopoietic stem cell transplantation (HCT). Clin Microbiol Infect 2012;18(suppl 7):53-67.

12. Cornely OA, Lasso M, Betts R, Klimko N, Vazquez J, Dobb G, et al. Caspofungin for the treatment of less common forms of invasive candidiasis. J Antimicrob Chemother 2007;60:363-369.

13. Legrand F, Lecuit M, Dupont B, Bellaton E, Huerre M, Rohrlich PS, et al. Adjuvant corticosteroid therapy for chronic disseminated candidiasis. Clin Infect Dis 2008;46:696-702. 